\title{
Alterstice
}

Revue internationale de la recherche interculturelle

International Journal of Intercultural Research

Revista International de la Investigacion Intercultural

\section{Formes, contenus et usages du témoignage dans les ONG d'aide internationale : la vérité à l'épreuve du marketing}

\section{Francine Saillant, Marie-Ève Drouin et Nathalie Gordon}

Volume 1, numéro 2, 2011

Droits humains et témoignages : l'épreuve de la culture

URI : https://id.erudit.org/iderudit/1077601ar

DOI : https://doi.org/10.7202/1077601ar

Aller au sommaire du numéro

Éditeur(s)

Alterstice

ISSN

1923-919X (numérique)

Découvrir la revue

Citer cet article

Saillant, F., Drouin, M.-È. \& Gordon, N. (2011). Formes, contenus et usages du témoignage dans les ONG d'aide internationale : la vérité à l'épreuve du marketing. Alterstice, 1(2), 35-46. https://doi.org/10.7202/1077601ar
Résumé de l'article

L'article propose un exposé et une analyse des contenus, des formes et des usages du témoignage dans les ONG d'aide internationale établies à Montréal. $\mathrm{Au}$ total, 17 ONG furent rencontrées lors de visites de sites, 34 entretiens semi-dirigés furent réalisés et 250 documents recueillis entre janvier 2009 et janvier 2010. La recherche a permis de préciser l'importance du témoignage dans les ONG, témoignages laissant peu de place à la voix des individus des populations aidées mais laissant une large place à leurs images. Toutefois, il est clair que ces messages, au vu de la manière dont ils sont construits et diffusés, proposent une vision des réalités du Sud aseptisée des maux qui affligent quotidiennement les populations. Les ONG privilédient une représentation des problèmes vécus par les populations aidées, ceci dans le contexte où ces dernières doivent faire face à de nombreuses contraintes de divers ordres, notamment politiques et culturelles. De cette étude se dégage une figure localisée du sujet de l'aide canadienne, " actif et engagé dans son développement ", une représentation qui s'inscrit en porte à faux de toute vision misérabiliste et sensationnaliste. (c) Francine Saillant, Marie-Ève Drouin et Nathalie Gordon, 2011
Ce document est protégé par la loi sur le droit d'auteur. L'utilisation des services d'Érudit (y compris la reproduction) est assujettie à sa politique d'utilisation que vous pouvez consulter en ligne.

https://apropos.erudit.org/fr/usagers/politique-dutilisation/ 


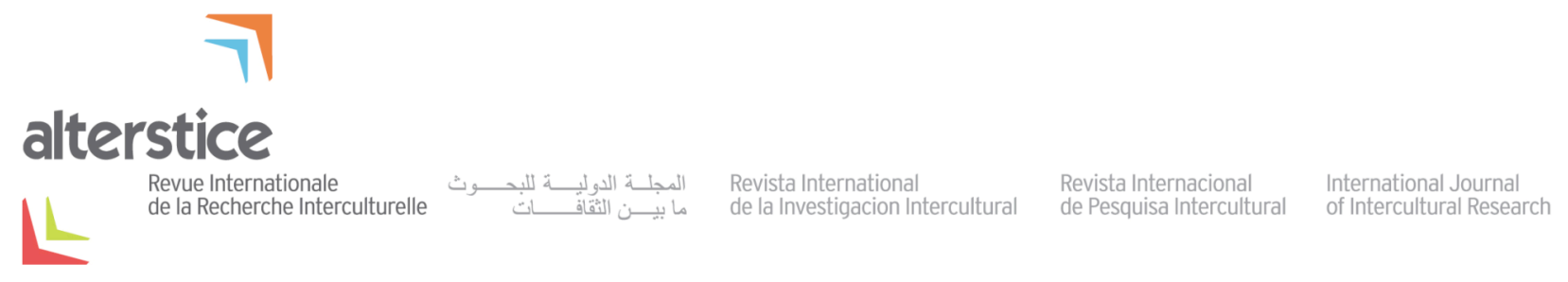

ARTICLE THÉMATIQUE

\section{Formes, contenus et usages du témoignage dans les ONG d'aide internationale : la vérité à l'épreuve du marketing}

Francine Saillant, Marie-Ève Drouin et Nathalie Gordon ${ }^{1}$

\section{Résumé}

L'article propose un exposé et une analyse des contenus, des formes et des usages du témoignage dans les ONG d'aide internationale établies à Montréal. Au total, 17 ONG furent rencontrées lors de visites de sites, 34 entretiens semi-dirigés furent réalisés et 250 documents recueillis entre janvier 2009 et janvier 2010. La recherche a permis de préciser l'importance du témoignage dans les ONG, témoignages laissant peu de place à la voix des individus des populations aidées mais laissant une large place à leurs images. Toutefois, il est clair que ces messages, au vu de la manière dont ils sont construits et diffusés, proposent une vision des réalités du Sud aseptisée des maux qui affligent quotidiennement les populations. Les ONG privilédient une représentation des problèmes vécus par les populations aidées, ceci dans le contexte où ces dernières doivent faire face à de nombreuses contraintes de divers ordres, notamment politiques et culturelles. De cette étude se dégage une figure localisée du sujet de l'aide canadienne, " actif et engagé dans son développement ", une représentation qui s'inscrit en porte à faux de toute vision misérabiliste et sensationnaliste.

\section{Rattachement des auteures}

${ }^{1}$ Département d'anthropologie et Centre interuniversitaire d’Études sur les Lettres, les Arts et les Traditions (CÉLAT), Université Laval, Québec, Canada

\section{Correspondance}

francine.saillant@ant.ulaval.ca

\section{Mots clés}

ONG; aide internationale; images; récits; témoignages

\section{Pour citer cet article :}

Saillant, F., Drouin, M.-E. et Gordon, N. (2011). Formes, contenus et usages témoignage dans les ONG d'aide internationale : la vérité à l'épreuve du marketing. Alterstice, 1(2), 35-46. 


\section{Introduction}

Le Québec et le reste du Canada fournissent une part significative de l'aide internationale par leur main-d'œuvre et leur présence dans le monde (Conoir et Verna, 2002). La plupart des ONG internationales ont pignon sur rue à Montréal et à Ottawa, reflétant les réalités bilingues et politiques du pays, et se dédoublant parfois dans l'une ou l'autre ville. Le Québec a aussi développé, depuis les années 1960, une importante tradition d'aide internationale dans les sillons du catholicisme de gauche et d'associations locales (Beaudet, 2009). La présence des ONG se fait plus forte au moment d'événements catastrophiques, par exemple le Tsunami en 2004 (Saillant, 2006), l'ouragan Kathrina en 2005 (Ethridge, 2006) et le récent tremblement de terre en Haïti en 2009. Au Canada comme ailleurs dans le monde, ces ONG jouent un rôle d'intermédiaire entre le grand public et les groupes qu'elles ciblent, par leurs pratiques de communication et une culture partagée de la promotion des droits humains ${ }^{1}$ et de la solidarité (Dauvin et Siméant, 2002). On peut dès lors se demander comment sont présentés au grand public québécois et canadien, par ces mêmes ONG, les groupes objets et acteurs de l'aide internationale, quelles sont leurs voix et leurs images et plus précisément leurs témoignages. Cette question peut paraître anodine, mais il faut se rappeler que les contenus médiatiques sont pour une partie importante du grand public canadien et québécois ce par quoi passe une part importante de sa connaissance sur les réalités vécues par les " groupes vulnérables » des pays du Sud ${ }^{2}$. De plus, les pays où sont implantés les centres administratifs des ONG internationales vont jusqu'à créer des profils de populations du Sud en fonction de paramètres de "désirabilité nationale ", toutes les causes ou populations n'étant pas égales aux yeux des gouvernements ou du grand public ${ }^{3}$. Les ONG jouent un rôle stratégique dans la diffusion de messages véhiculant des contenus projetant l'altérité, messages qui intéressent ceux qui réfléchissent aux cadres de l'interculturalité à l'échelle des nations plutôt qu'à celle des interactions. Ce qui signifie que, dans le cas que nous examinons ici, les relations interculturelles entre populations du Nord et du Sud sont en quelque sorte filtrées par les représentations sociales que projettent les ONG par le biais de leurs différents moyens de communication.

Un examen attentif des formes, contenus et usages du témoignage dans les ONG à caractère international établies à Montréal, qu'elles soient canadiennes, québécoises ou étrangères ${ }^{4}$, nous amènera à traduire les formes de représentation de l'altérité et de processus d'altérisation qui sont en jeu.

\section{Le témoignage}

L'étude du témoignage en circulation dans l'espace public à travers les messages des ONG suppose une certaine clarification conceptuelle. En effet, à ce jour, la notion de témoignage a été peu problématisée dans les sciences sociales, quoique ce soit d'abord et avant tout « une forme de récit public impliquant une adresse de la part d'une victime à un tiers quant à un tort subi ", et qu' elle ait connu un certain essor depuis la publication du livre de Primo Levi Si c'est un homme (1947), surtout depuis la traduction en français de cet ouvrage et des œuvres complètes de son auteur (2005). Outre les efforts de définition et de clarification conceptuelle proposés par Dulong (1998) dont nous nous inspirons en partie, c'est en effet toute une littérature qui a vu le jour à propos de la Shoah et des génocides et dont les littéraires, mais aussi les historiens, sociologues et anthropologues, ont été les auteurs (Coquio, 2003; Chiantaretto et Robin, 2003). Cet accent sur la Shoah (Wieviorka, 2002; Truchon, article dans ce numéro), prophétique quant aux génocides qui suivirent, a amené les auteurs à centrer leurs propos sur la mémoire et le devoir de mémoire (Chaumont, 2002; Erner, 2006; Todorov, 2004), le témoignage devenant de la sorte assujetti aux conditions mêmes de la transmission mémorielle, aux relations entre le passé, fût-il récent, et les impératifs du présent (Ricœur, 2000).

\footnotetext{
${ }^{1}$ L'usage de cette expression est expliqué dans le texte introductif au numéro (Saillant, 2011).

${ }^{2}$ L'expression Sud renvoie à l'ensemble des pays dits en développement (Deep South) et non pas à une région géographique précise.

${ }^{3}$ Ainsi le tremblement de terre survenu au Pakistan en 2010 a-t-il suscité peu d'émoi comparativement à celui d'Haïti la même année.

${ }^{4}$ Les résultats sont issus de la recherche Droits humains et témoignages dans les organisations humanitaires au Canada et au Québec, financée par le FQRSC (2007-2010).
} 
Le témoignage a aussi été étudié plus directement dans les ONG par un certain nombre d'auteurs qui ont cherché à connaître ses formes, ses contenus et ses usages. Ces travaux, peu nombreux, ont mis l'accent sur l'instrumentalisation de la parole d'individus des populations étudiées aux fins politiques ou financières des ONG (Maalki, 1995 et 2002; Fassin et Rechtman, 2007; McLagan 2003; Gregory, 2006; Hastrup, 2003). On a peu tenu compte, sauf exception (Saillant, Paumier et Richardson, 2005; Saillant, 2006, 2007a, 2007b et 2007c), des formats technologiques liés à la multiplication, à la répétition et à la large diffusion des contenus des messages, notamment sur internet, ce qui contribue du coup à construire, dans l'espace public, des figures spécifiques de l'altérité et des processus d'altérisation (Spivak, 2009), de même qu'à "détextualiser » les témoignages en donnant une importance de plus en plus grande aux images des populations et à une part réduite du contexte de leurs expériences. Le témoignage, pour cette raison, prend de plus en plus un caractère performatif, et agit plutôt que textuel et étroitement narratif (Saillant et Boudreault-Fournier, 2011). II faut aussi rappeler que les ONG, avec l'aide de leurs services de marketing, se sont mises à l'heure de la popularité des récits personnels et émotionnels (Salmon, 2009) dans les médias de masse, à travers des techniques associées au storytelling ou aux diverses formes de télé-réalité (Charbonneau, 2011). Elles se doivent, sans nécessairement les copier, atteindre un public parfois fatigué des messages compassionnels (Boltanski, 1993), mais toujours épris d'émotions fortes, émotions déployées dans un espace public qui marche plus que jamais au partage de l'intime.

Une autre approche du témoignage, qui a pris un certain essor en anthropologie, a été celle de la souffrance sociale (Fassin, 2004a, 2004b, 2004c et 2007; Das, Kleinman, Lock, Ramphele et Reynolds, 2001; Das, Kleinman, Ramphele et Reynolds, 2000; Kleinman, Das et Lock, 1997; Blais, 2008). Les contextes de production des récits déployés au nom du "droit d'avoir des droits ", au sens de l'expression de Rancière (2005), ont été à l'origine d'un certain nombre d'études (Saillant et Truchon 2008; Saillant, 2011). Pour ces auteurs, le témoignage produit au sein des ONG se présente comme un récit doublement contraint par la culture nationale ou régionale des sujets témoins et par celle des ONG elles-mêmes.

Parmi toutes ces études, il n'existe pas à notre de connaissance de travaux orientés sur le témoignage ayant pris pour terrain les ONG établies au Québec ou ailleurs au Canada. Ces constats amènent à chercher à comprendre les particularités des situations de révélation, de communication et de circulation des témoignages, ainsi qu'à tenter d'identifier qui parle au nom de qui, et de quoi, comment et pourquoi depuis les ONG. De telles questions ne sauraient passer sous silence que ces « voix et images du Sud » que transmettent les ONG sont autant de paroles et d'icônes en circulation dans le monde et dans les pays du Nord qui imprègnent les imaginaires à propos d’altérités convenues.

\section{Méthodologie}

Répondre à de telles questions nous oblige à préciser davantage le sens de la notion de témoignage. On ne saurait d'abord réduire le témoignage à la situation juridique devant un tribunal, par exemple à celle vécue par les demandeurs d'asile devant les instances gouvernementales de l'immigration, ou encore à celle de victimes et de criminels devant les tribunaux internationaux, comme c'est le cas au Tribunal pénal international (TPI) (Saillant et Truchon, 2008). Le témoignage appartient dans ce cas au registre de la preuve (Kilani, 2009; Hastrup 2001a et 2001b) et de la vérité à établir devant des droits bafoués, puisqu'il s'agit de trancher, d'identifier victimes et bourreaux, de sanctionner, de redresser un tort. Le témoignage doit pourtant selon nous s'ouvrir à d'autres contextes associés aux manquements aux droits, par exemple ceux que révèlent par leurs pratiques les ONG. Sur le plan empirique, le témoignage engloberait ainsi l’ensemble des récits et images énoncés, produits puis diffusés dans le grand public à travers différents formats et concernant des situations d'aide ou des exactions de droits légitimant l'action des ONG. Ces récits sont autant ceux des destinataires de l'aide que ceux des acteurs des organisations auxquels ils se réfèrent.

Nous estimions en 2007 à une vingtaine le nombre d'ONG d'aide internationale établies sur le territoire montréalais. Une tournée fut entreprise auprès de ces dernières. Lors de chaque visite, une combinaison de techniques fut utilisée: entrevues semi-dirigées enregistrées, entrevues informelles, mise en contact avec des pratiques en cours ayant directement trait au témoignage, collecte et examen de documents produits par les organismes et commentés devant nous, tout cela variant en quantité selon les possibilités de l'organisme. Des 
personnes occupant diverses fonctions et engagées dans les pratiques relatives au témoignage furent rencontrées. Lors de chaque visite, le temps de la rencontre a varié entre deux heures et une journée. Les personnes rencontrées ont pu s'exprimer sur les pratiques du témoignage au sein de leur organisme, présenter celles-ci et les commenter en partageant des informations à propos de documents divers produits par les ONG et susceptibles de contenir des témoignages. Elles ont la plupart du temps prêté ou donné ce matériel lorsque nous l'avons considéré comme représentatif des formats, des usages ou des contenus. Enfin, les personnes rencontrées ont pu aussi partager leurs questionnements courants en lien avec l'éthique des groupes vulnérables, les pratiques de développement et les pratiques humanitaire concernant le témoignage. Ces visites se firent pour la plupart à deux. Au total, ce sont 17 ONG qui ont été visitées. Au sein de ces ONG, ce sont $34^{5}$ personnes qui ont été rencontrées et qui ont accepté les entrevues semi-dirigées. La moitié d'entre elles occupaient des fonctions de directeur ou chef de service du marketing et des communications; l'autre moitié, de chef de programme ou de secteur, d'agent de communication ou de simple coopérant. Au cours de ces visites, 250 documents ont été recueillis, dont des publireportages, des émissions de radio ou de télévision, des bulletins électroniques, des documents de campagnes de financement, des outils promotionnels, des vidéos et des rapports annuels. Nous avons sélectionné parmi ce matériel celui dont nous avions discuté avec le personnel des ONG et les exemples typiques que ce dernier a jugé représentatif de son organisation ${ }^{6}$.

L'analyse qualitative de contenu des entrevues et des documents a été développée de façon à faire ressortir

- I'histoire, les mandats et les structures des organisations,

- les positions quant aux valeurs face aux droits et au témoignage,

- les formats du témoignage,

- les contenus à valoriser ou à éviter,

- les débats suscités par les choix à effectuer,

- les contraintes liées au témoignage.

La prochaine section présente les résultats en fonction de l'importance accordée au témoignage et aux droits dans les organisations, les usages des témoignages et l'identité des témoins, les contenus valorisés ou écartés et enfin leurs contraintes structurelles. La conclusion apportera des réflexions complémentaires sur l'altérisation par le témoignage et sur la notion même de témoignage.

\section{Résultats}

Importance du témoignage et des droits dans les ONG

Le témoignage apparaît central dans la très grande majorité des ONG visitées. L'abondance et la diversité du matériel fourni indiquent déjà la place qu'on leur accorde. Mais à quoi servent véritablement les témoignages? D'abord à la sensibilisation : les ONG se doivent de diffuser au grand public leur raison d'être et surtout les causes de leurs engagements. Pourquoi agir sur tel ou tel pays, pourquoi concentrer ses énergies sur le genre, le handicap ou la faim? Comment justifier? La sensibilisation, ou si l'on préfère l'éducation du public, sert directement ce besoin. En sensibilisant le grand public, les ONG diffusent leurs perspectives sur les droits, le développement et la solidarité internationale, elles se forgent un espace dans les représentations collectives quant aux institutions de la société civile « qui comptent » en tant qu'actrices à l'œuvre et d'envergure; enfin, elles trouvent de cette façon le moyen de présenter leurs besoins et leurs nécessités, en termes de main d'œuvre, de collaborations et de finances. Par ce moyen, elles favorisent aussi des gestes de solidarité qui se traduisent potentiellement en dons, que ce geste suive directement ou non une campagne de financement. Le témoignage, que certains traduisent par le mot

\footnotetext{
${ }^{5}$ Une cinquantaine d'entrevues informelles ont aussi été effectuées avec divers agents des ONG rencontrés lors de nos visites. ${ }^{6}$ L'ensemble de ce matériel n'a pas fait l'objet d'une analyse systématique compte tenu des moyens limités de la recherche. Le choix de concentrer notre attention sur des formats qui ont été discutés lors de nos visites s'est toutefois avéré suffisant compte tenu du caractère parfois répétitif des messages d'un format à l'autre et même d'une ONG à l'autre.
} 
plaidoyer ${ }^{7}$, est considéré par plusieurs comme exceptionnel puisqu'il donne chair au programme et à des réalités autrement plus complexes à traduire. Le fait de traduire, par des récits et des images, les réalités des expériences de développement et d'aide humanitaire offre une trame sensible de connexion entre le Nord et le Sud, les coopérants et les pays donateurs. II faut, dit-on, "des sources d'inspiration pour toucher le grand public ». II faut "plus que du discours" mais "montrer des réalités sensibles ", créer des ouvertures pour qu'une certaine identification aux causes et aux populations du Sud puissent "passer». On veut quitter l'espace de la communication froide des grands médias, ou encore leur caractère sensationnaliste, montrer des réalités qui permettent à la fois l'ouverture aux autres et leur connaissance et ainsi faire saisir que l'aide "peut faire la différence ». Les témoignages, c'est ici le moyen que les ONG utilisent pour aller à la rencontre des gens, populations du Sud lorsqu'ils sont recueillis, populations du Nord lorsqu'ils sont transmis. Le témoignage utilisé de cette façon est sans doute le cas le plus fréquent et le plus répandu au sein des diverses ONG rencontrées. Deux autres fonctions lui sont dévolues : il est souvent une source d'inspiration à la base de la construction des cadres de référence, ou encore il sert à développer les activités éducatives et d'advocacy ${ }^{8}$ afin de favoriser la transmission de valeurs démocratiques.

Alors que le témoignage est de toute première importance pour les ONG, qu'en est-il des droits humains ? Dans la majorité des ONG rencontrées, le thème des droits humains est transversal pour une raison bien simple : aucune des ONG rencontrées ne se présente comme une ONG d'advocacy ${ }^{9}$. Les droits humains font partie, pour la plupart des ONG, des principes d'action, mais on n'y travaille pas obligatoirement de façon spécifique ou directe. Ce qui veut par exemple dire qu'agir contre la faim est une manière concrète de se conformer à l'article 25 de la Déclaration universelle des droits de l'homme (DUDH) sans pour autant se traduire en action d'advocacy sur la question. Aux yeux des ONG, travailler sur les droits humains, c'est concentrer son attention et son énergie sur leur application et sur les acteurs gouvernementaux et supranationaux dont c'est la spécialité. Le témoignage direct lié aux manquements aux droits est souvent jugé comme risqué, car il peut mettre à l'épreuve des pays autoritaires : il n'est donc pas toujours souhaitable ou désirable, et peut même se retourner contre les témoins. Ces circonstances incitent à la prudence.Témoins et témoignages

Qui sont ceux qui agissent en tant que témoins dans les récits des ONG qui circulent vers le grand public? De façon très majoritaire, le personnel des ONG lui-même. On se réfère ici à des volontaires qui rapportent leurs histoires personnelles et leurs photos et qui les placent dans des bulletins, des blogues ou en balado; à des chargés de projets qui rencontrent des personnes susceptibles de témoigner de situations et d'actions. On se réfère aussi à des personnes qui, dûment formées, sont envoyées sur place pour documenter un cas ou une expérience et permettent de préciser une action à venir ou d'évaluer celle qui est en cours : on a alors besoin de savoir comment les populations concernées par l'aide se situent, évaluent leurs besoins et les projets. Dans l'écrasante majorité des cas, ce sont les bénévoles et les coopérants internationaux ou locaux (d’abord les premiers, ensuite les seconds) qui effectuent ces témoignages, donc qui se font les traducteurs des expériences de ceux qu'ils aident. Beaucoup plus rarement, les personnes du Sud et les populations aidées sont les auteurs de leurs propres témoignages. Si la voix du Sud passe peu par la parole directe, écrite ou enregistrée, des populations concernées, lorsqu'il est question d'images, la situation s'inverse. En effet, ce sont en premier lieu des individus des populations aidées qui se retrouvent sur les affiches, les publipostages, les sites web, puis les coopérants du Sud et en dernier lieu les coopérants du Nord.

\footnotetext{
${ }^{7}$ Le plaidoyer est dans le domaine du droit le discours prononcé par un avocat dans le but de défendre une cause face à un juge. Plus largement, toute personne peut effectuer un plaidoyer dans le but de défendre une cause ou une idée. Le témoignage n'est pas nécessairement un plaidoyer car il peut se présenter comme un simple récit d'expérience sans nécessairement être structuré selon une rhétorique de défense. Les ONG ont toutefois lors des entrevues souvent fait équivaloir témoignage et plaidoyer.

${ }^{8}$ L'advovacy, différent du témoignage, est une forme de pratique active d'influence issue de la société civile et effectuée par un individu ou un groupe d'intérêt dans le but d'amener un gouvernement ou des décideurs à transformer une réalité, par exemple en matière de justice.

${ }^{9}$ Ce qui serait le cas d'Amnistie internationale, une ONG dont la pratique est basée à la fois sur l'advocacy et l'usage du témoignage. Cette ONG n'intervient pas directement dans les pays du Sud comme le fait par exemple Médecins sans Frontières, elle n'entrait donc pas dans l'échantillon de départ.
} 
Les témoignages eux-mêmes et leurs contenus ne sont à peu près jamais spontanés, mais plutôt le produit de commandes. Les ONG sont les demandeuses, et elles souhaitent des contenus précis à partir des thèmes, des projets, des contextes sur lesquels des élaborations et des illustrations sont nécessaires. On se réfère alors à ce qui est vécu habituellement par le plus grand nombre, ou aux situations courantes des programmes. On ne veut pas mettre l'accent sur les situations exceptionnelles, même si elles sont « vendeuses » pour le grand public. La plupart du temps, les contenus sont choisis par le service marketing des ONG, par un département connexe ou, dans le cas où ces services sont inexistants, par la direction elle-même.

On peut en dernier lieu se demander ce qui est reconnu comme témoignage à travers la multiplicité des communications allant du Sud vers le Nord et pouvant servir de matériel pour les communications publiques. Qu'est-ce qu'il faut dire et montrer, ne pas dire ou ne pas montrer, et quelles contraintes pèsent sur les témoins et leurs témoignages?

\section{De ce qu'il est préférable de dire}

Les ONG ont insisté sur l'importance de témoigner de leur travail, de leur présence et de leurs actions sur le terrain. II faut ainsi, préférablement, montrer des personnes qui sont actives, volontaires dans leurs projets, dynamiques. II faut aussi montrer des résultats et surtout des résultats positifs. Cela peut par exemple se faire en illustrant l'avant et l'après d'un projet. Ainsi, on ne veut pas nécessairement insister sur la souffrance des populations, mais au contraire sur leur puissance d'agir et sur les histoires qui marchent, les projets exemplaires qui réussissent. L'idée est ici de communiquer clairement ce qui se fait dans la coopération canadienne, afin que le public et les destinataires saisissent ses orientations, sa structure. On veut de ce fait faire circuler les valeurs des organisations, et c'est de cette façon que certaines histoires seront retenues plutôt que d'autres. Ces valeurs peuvent être par exemple l'éducation, le logement, la santé, le syndicalisme. On choisira alors des paroles et des images ayant ces valeurs comme référence. II faut ainsi démontrer la volonté des coopérants, que l'on puisse voir ces derniers en action sur le terrain, encore une fois au sein de projets "qui donnent des résultats » aux yeux des groupes desservis et des ONG elles-mêmes, résultats "lisibles " par ceux à qui s'adressent les témoignages et les produits des communications. Les ONG cherchent, il faut le souligner, à lutter contre des préjugés qui circulent dans les pays destinataires et dans certaines couches de leur population, ceux qui laissent croire l'aide internationale inutile : les coopérants ne feraient de ce point de vue que perdre leur temps à l'étranger. Ces préjugés, bien sûr, vont à l'encontre des points de vue des ONG.

Du côté des volontaires et des coopérants, il est pensable pour eux de valoriser leur expérience à travers leurs images et leurs récits, en faisant place à l'idée selon laquelle aller à l'étranger, s'adapter à une autre culture, faire en sorte que son travail soit utile, est quelque chose de difficile, qui ne va pas de soi, et qui va à l'encontre des constructions négatives accolées parfois à leurs identités. Les ONG considèrent d'ailleurs que les meilleurs témoins sont finalement ces personnes, placées entre l'organisation et les populations desservies, dans l'action avec ces dernières, au plus près du travail concret, par exemple des chefs de projets locaux ou des coopérants sur le terrain, ce qui ne sera pas nécessairement le cas des chefs de programmes et des fonctionnaires dans les bureaux des pays donateurs.

Volontaires et coopérants agissent au premier plan des messages, surtout ceux en direction du Nord. Les témoignages retenus vont concerner d'abord et avant tout les problèmes locaux : leur ampleur, leur gravité, leur représentativité. L'ampleur d'une situation, cela peut renvoyer à un désastre écologique assorti de témoignages sur la distribution d'eau dans les communautés touchées. La gravité, surtout hors de l'urgence, n'est pas évidente : comment en effet expliquer que la malnutrition chronique est un problème aussi grave que celui de la violence en situation de guerre? La représentativité ne signifie pas nécessairement se centrer sur les cas extrêmes, mais aussi sur les cas les plus courants: loin de l'urgence et plus près des réalités quotidiennes, hors des grandes crises et catastrophes. La sobriété des messages plutôt que la spectacularisation est de mise. C'est ainsi que l'on pense que l'un des défis de la " pédagogie du témoignage " (notre expression) est celui de faire valoir ce qui paraît à première vue le moins important. Le témoignage d'une seule personne peut renvoyer à des centaines de situations identiques dans une localité ou même au-delà. Ainsi, plutôt que de montrer la gravité d’une blessure de guerre, on 
choisira une scène de rééducation, jugée plus positive, et qui met en valeur le courage des uns et l'action des autres.

Cela nous amène vers le traitement des contextes, puisque ces derniers appellent à être définis afin que les actions des ONG prennent sens aux yeux de ceux qui sont loin de l'aide internationale. Certaines images et paroles vont ainsi laisser place aux bénéficiaires de l'aide et à leur environnement, par exemple leur famille, leur habitat, leur travail. On peut vouloir montrer le résultat plutôt que le besoin : par exemple ne pas laisser passer un visage triste, mais plutôt une maison à construire, ce qui projette l'attention sur l'avenir et sur l'action vers cet avenir. Cette position idéologique dans le choix des images et des paroles transmises est extrêmement névralgique : sans cacher la misère, il faut, selon les ONG, s'assurer que le message principal transmis à propos de leurs actions et des contextes des réalités du Sud soit celui du potentiel et de l'espoir pour une véritable transformation.

Nous l'avons souligné, le témoignage des communautés locales est plus rare dans les communications des ONG. Certaines ONG le valorisent, et dans ce cas elles choisissent ce qu'elles appellent le témoignage collectif : celui qui va au-delà de l'expérience personnelle et se hisse au rang de la réalité vécue par l'ensemble d'une communauté. Celui qui permet à ceux qui n'ont pas de voix de prendre une place au sein d'un projet. Cela n'est pas si facile, car on a besoin alors de leaders capables de le faire: les enfants, les personnes les moins scolarisées ont plus de difficulté dans ces situations, à quoi s’ajoutent les obstacles liés à la langue.

Parce que les ONG rencontrées ne sont pas des ONG orientées exclusivement sur l'advocacy, il est certain que cette part de leur activité devient dans les communications un contenu moins fréquent, mais pas pour autant moins important. Dénoncer signifie rendre publics les manquements aux droits, par exemple aux accords internationaux. Lorsque Handicap International s'engage dans la dénonciation de l'usage des bombes à munition et de leurs effets sur les populations locales, elle le fait en cherchant à contribuer à une action plus efficace de la part des pays qui ont signé la convention sur les bombes antipersonnel et de la part des acteurs sur le terrain. La promotion du respect des droits suit donc cette logique, mais une attention très particulière est accordée au fait que toute image et toute parole retenue doit se faire dans le respect des droits de ceux que l'on prétend défendre. Par exemple, montrer une femme ou en enfant sera fait en respectant la vie privée, la dignité des personnes et leur volonté de témoigner.

Un dernier aspect du traitement des témoignages est celui qui concerne les communications elles-mêmes. Chaque ONG a son style, son branding comme elles disent. Les structures des communications publiques des ONG vont ainsi peser sur les choix et le traitement des témoignages. D'abord, toutes insistent sur l'évitement d'une approche dite "marketing ": éviter le sensationnalisme et la spectacularisation est un principe organisateur. On préfère, dit-on, éduquer plutôt que séduire, ne pas choquer ou violenter le lecteur, se démarquer des médias de masse, décidément opter pour le modèle contre-identificatoire. Les témoignages retenus devront être empreints de sensibilité afin de rejoindre le grand public, mais de façon subtile: par exemple, laisser passer les rêves des populations desservies, leurs luttes, leurs espoirs les plus profonds. On ne montrera pas, en corollaire, les photos de violence extrême, de femmes violées, d'enfants séquestrés. Le danger est d'abord que les ONG elles-mêmes manquent aux droits de ces personnes en diffusant des images et des paroles qui pourraient être reçues comme dégradantes. Le grand public risque aussi de décrocher de l'importance des actions des ONG pour se concentrer sur le misérabilisme. Or le misérabilisme est l'ennemi numéro un des ONG qui, entre elles, se jugent parfois sévèrement si certaines vont «trop loin » dans leurs messages. II faut, aux yeux des ONG, pouvoir être à l'aise de montrer aux populations locales ce que l'on diffuse à leur propos, valoriser le life saving work, c'est-à-dire «le travail qui change la vie ». La sensibilité des pays destinataires et leur culture doivent de ce point de vue compter largement dans les choix. En contexte canadien et québécois, on mettra ainsi l'accent sur des réalités qui font sens dans cette même région du monde, par exemple celles qui sont liées à l'égalité homme-femme. Le grand public au Québec et dans le reste du Canada est également sensible au pragmatisme : encore là, la question de l'action et des résultats, ainsi que de leur importance dans les messages, reste centrale. Par exemple, à l'UNICEF on affirme que la majorité des messages concernant les enfants les montrent comme souriants et ayant eu accès à des services. 


\section{De ce qu'il ne faut pas dire}

Des contextes spécifiques amènent les ONG à taire certains témoignages, et ce, dans trois catégories de circonstances: lorsque le témoignage prend pour objet les organisations d'aide, les populations locales, les contraintes politiques et de sécurité des pays destinataires des actions sur le terrain.

Les ONG considèrent que le principe de non-nuisance est primordial lorsque les communications prennent les organisations elles-mêmes comme centre de leurs contenus, par exemple lorsqu'elles se présentent au grand public avec leur branding. D'abord ne pas nuire à un projet : cela peut vouloir dire éviter de créer des problèmes avec les directions des ONG ou avec les donateurs, de manière à ce que l'existence d'un projet ne soit pas compromise par des coupures de fonds. Une organisation catholique peut ainsi se montrer plus sensible à des projets non conformes aux orientations de l'Église et des irritants se transforment alors en nuisances. II ne faut pas non plus nuire à la réputation d'une ONG face au grand public, qui a le pouvoir de réagir par la tiédeur aux levées de fonds. Ce sont aussi les employés des ONG qui ne sauraient être visés par des propos disqualifiant leur travail ou leur réputation, ou encore les plaçant eux-mêmes en situation de vulnérabilité. Pour cela, on préférera que les témoignages soient ceux des chefs de projets, et non nécessairement ceux des intervenants terrain, plus fragiles et plus à risque, en particulier lorsque les propos sont susceptibles d'être compris comme une critique des gouvernements ou des instances locales. Le choix de valeurs rétrogrades (ex. : racistes ou sexistes) serait une autre manière de nuire à l'ONG en se plaçant à contre-courant des valeurs des donateurs qu'on suppose enlignées sur celles des ONG. Un dernier aspect touche la très grande difficulté à parler de ce qui ne marche pas dans les actions et les résultats sur le terrain ou dans l'acheminement de l'aide, une question très sensible. Les centaines et centaines de projets des ONG ne peuvent évidemment pas tous s'avérer des opérations à succès, surtout lorsqu'ils sont observés à court terme. Leurs problèmes particuliers ressortent bien entendu des évaluations ponctuelles ou encore des rapports de fin de projet. Cependant, on ne trouve jamais de propos sur les projets boîteux ou problématiques dans les communications au grand public: il y aurait là nuisance et effets potentiellement dévastateurs pour ces projets, les coopérants et les populations locales.

Les propos centrés sur les populations locales sont aussi à considérer avec prudence. Le misérabilisme évoqué précédemment est une nuisance : on ne veut jamais réduire les individus des populations aidées à leur dimension de victimes. C'est pourquoi les images et les propos pessimistes seront écartés : quoique les situations dramatiques existent, on veut toujours montrer ce que les gens font déjà pour s'en sortir, ce que les ONG poursuivent comme objectifs de concert avec les populations locales. Montrer des individus en situation d'impuissance est nuisible : par exemple, montrer une personne qui aurait subi une agression en situation de guerre signifierait exhiber son intimité et sa vulnérabilité, un geste contraire à l'esprit du travail des ONG qui, justement, luttent contre la vulnérabilité. Enfin, certains témoignages seront rendus impossibles parce que d'elles-mêmes les populations locales se taisent et refusent de communiquer certaines situations par peur d'être en conflit avec leur gouvernement ou encore de se faire couper des services par les ONG. Dans le second cas, cela veut dire que des propos plus critiques à l'endroit des ONG sont difficilement exprimés et, s'ils le sont, on peut vite saisir qu'ils ne seront pas communiqués au grand public.

Le troisième thème qui regroupe les propos des ONG sur les contenus à taire ou éviter est celui du politique et de la sécurité. Cette question est devenue de plus en plus centrale depuis que les politiques de sécurité se sont faites plus serrées à partir du 11 septembre 2001. On évite d'abord et avant tout de critiquer les gouvernements en place afin de ne pas nuire aux populations locales et aux ONG elles-mêmes et à leurs coopérants. Les communications évoqueront les problèmes (ex. : conditions de vie) sans nécessairement viser les gouvernements en place (ex. : politiques nationales). Un chef sanguinaire d'un gouvernement d'un pays destinataire n'apparaîtra pas dans les messages, pas plus que celui d'un gouvernement conservateur d'un pays donateur. On craindra, sans doute avec raison, plus de cruauté de l'un ou plus de radinerie de l'autre. On ne cherchera donc pas à susciter un débat social, ni dans les pays donateurs ni dans les pays destinataires, débat qui ferait trop de bruit et « créerait de la polémique inutile ". Enfin, on ne cherchera pas non plus à culpabiliser les citoyens des pays donateurs: il ne s'agit pas de conditionner leur don aux nombres de morts évités mais de les éduquer à la solidarité, ce qui est considéré comme différent. 


\section{Les contraintes du témoignage}

Les politiques des gouvernements des pays liés aux circuits de l'aide jouent pour beaucoup dans le style des communications et des témoignages, qui de ce fait devront prendre obligatoirement certaines formes. Le conservatisme du gouvernement donateur, par exemple celui du gouvernement canadien actuel qui est peu généreux à l'endroit de l'aide internationale, influe sur les choix des communications car le couperet tombe rapidement. Une organisation qui ne se conforme pas suffisamment aux orientations du gouvernement en place verra les fonds en provenance de l'Agence canadienne de développement international (ACDI) diminués ou carrément coupés. Lors de nos visites, certaines organisations étaient ainsi extrêmement fragilisées par ces politiques conservatrices et menacées quant à leur survie. Les politiques des gouvernements des pays destinataires de l'aide sont également contraignantes : certains gouvernements locaux vont parfois jusqu'à contrôler toutes les communications issues des ONG, et ils se font plus lents pour les délais de visa ou encore pour rendre le matériel d'aide disponible depuis les douanes. Les expulsions des travailleurs de l'aide sont des cas qui, sans être réguliers, font partie du paysage. Ainsi, ces gouvernements autoritaires ont besoin de cette aide internationale conditionnelle à leur image extérieure, mais ils ne la reçoivent pas sans résistance ou sans leurs propres conditions, notamment celle de ne pas se retrouver salis sur la place publique.

Les pays où sévit une violence structurelle sont aussi enclins à contrôler fortement les ONG et leurs messages. Ce fut par exemple largement le cas en Amérique latine lors des massacres qui survenaient à intervalle régulier à l'époque des dictatures. Les ONG dénonçaient ces dernières, mais cachaient les noms des victimes de peur des représailles. Les situations de guerre où l'intervention des ONG mêle malencontreusement aide humanitaire sur le long terme et aide en situation d'urgence sont de plus en plus discutées, car ce mélange pervertit l'aide et confond les intentions et les actions de toutes les parties. Malgré ces critiques, dans certaines situations, les ONG se doivent de laisser le contrôle des communications aux militaires afin de ne pas contribuer à brouiller les messages, ce qui alimente du coup la polémique sur la nature des interventions des ONG, jugées alors trop collées sur les actions des belligérants ${ }^{10}$.

Les grands médias sont vus comme une contrainte supplémentaire : en effet, les ONG sont très conscientes du fait que leurs propos sont peu populaires dans les talk shows ou les grandes émissions d'actualité, pas assez glamour et porteurs de messages trop complexes, qui s'insèrent difficilement entre un humoriste et un joueur de hockey. Les ONG considèrent leurs contenus de portée surtout éducative, plutôt que strictement informative : elles se distancient, répètent-elles, des médias ordinaires. Les débats idéologiques seront donc évités devant les grands médias, car on soupçonne ces derniers de manichéisme: par exemple, un débat public sur l'efficacité de l'aide internationale ne se fera pas ou sera évité de crainte que ce soit mal traité et interprété.

Les contraintes des témoignages relèvent aussi des formats prédéfinis des communications. En effet, chaque ONG possède son modèle de communication lié à une image publique issue des réflexions des professionnels du marketing, comme on le fait dans les entreprises. Par exemple, certains mots seront évités, tels que charité ou religion, et certaines façons de faire seront privilégiées : dire merci ne se fera jamais au nom d'un destinataire, mais au nom de l'ONG. Les contraintes culturelles des pays donateurs jouent aussi sur les contenus: au Québec, la philanthropie laïque a meilleure presse que du côté du Canada anglais, où les messages à connotation religieuse passeront plus facilement. On en tiendra compte localement au Québec, mais cela deviendra problématique si les communications viennent de Toronto et doivent être reprises sans aucun filtre... Ces sensibilités différentes entre pays donateurs expliquent aussi les différences entre des messages d'une même ONG provenant de sièges sociaux situés dans diverses capitales du monde: les ONG procèdent lorsqu'elles le peuvent à une adaptation locale de leurs messages afin de s'assurer d'une bonne réception dans les pays donateurs. Dans le cas de destinataires très ciblés, prenons pour exemple le monde des affaires, un message trop féministe sera évité, car on prend en compte la composition à dominante masculine des directions d'entreprise. Les ONG sont conscientes qu'elles ne peuvent toucher tout le monde en même temps et que, même si une partie de la population des pays donateurs pourrait être plus sensible aux questions de violence et de pauvreté, une autre partie ne le sera pas du tout.

\footnotetext{
${ }^{10}$ La question de l'humanitaire et de ses relations avec le militaire mériterait à elle seule un développement particulier. Voir à ce sujet Fassin et Pandolfi (2010).
} 


\section{Conclusion}

Comme on vient de le voir, le témoignage dans les organisations d'aide internationale n'est pas une pièce négligeable. Il touche de nombreux aspects des activités, allant de la collecte des données de terrain à des fins de préparation ou d'évaluation en passant par des formes d'échange autour du vécu des programmes à travers les médias sociaux et par diverses animations éducatives et promotionnelles. Le développement des technologies de communication a rendu la diffusion de ces témoignages incontournables, parties intrinsèques d'un monde truffé d'histoires personnelles et de " vécu » à consommer, ainsi que de preuves par l'image. Les ONG n'y échappent pas, tout en en faisant vertement la critique, et avec raison. Désireuses d'atteindre leur public là où il se trouve, elles n'hésitent pas devant les twitter et autres médias sociaux, tout en ne délaissant pas leurs moyens traditionnels de diffusion et en les diversifiant.

Le témoignage sert surtout d'illustration concrète et sensible des réalités des populations destinataires de l'aide vers les pays donateurs, alliant alors éducation, sensibilisation, diffusion des valeurs de la solidarité et enfin activités de financement. L'un des résultats les plus prégnants de cette recherche est, d'une part, la rareté des témoignages issus des pays destinataires et énoncés directement par des membres des populations bénéficiaires de l'aide dans les messages en direction des pays donateurs et, d'autre part, l'importance des images qui par ailleurs représentent ces populations. Ce qui amène à comprendre que la majorité des messages en provenance des ONG d'aide internationale font peu de place aux bénéficiaires de l'aide et à la valeur de leurs témoignages par la parole, leur préférant ceux de leurs intermédiaires, chefs de programmes ou encore intervenants sur le terrain. $\mathrm{Au}$ contraire, les images qui accompagnent ces messages font une large place aux bénéficiaires de l'aide, les présentant selon des standards assez partagés d'une ONG à l'autre. Ils sont présentés comme actifs, souriants et volontaires : ils prennent part à "leur » développement, ils sont engagés dans des actions "transformatrices ", ils sont libérés en partie du "poids du malheur " qui a justifié l'aide. Le sujet local (canadien, québécois) de l'aide internationale, muet, laisse parler pour lui les images du Sud qu'il représente, images qui laissent la souffrance, les droits lésés et la violence structurelle hors champ. Il est certain que les ONG expliquent cette situation de nombreuses manières: respect et dignité des bénéficiaires de l'aide, chasse au misérabilisme et au sensationnalisme, prise en compte de la sensibilité du public, contraintes politiques et culturelles de divers ordres. Toutefois, il est clair que la manière dont ces messages sont construits et diffusés conduit à une vision des réalités du Sud aseptisée des maux qui affligent quotidiennement les populations.

Ces images sont aussi, comme d'autres auteurs l'ont souligné avant nous (Ethridge, 2006; Gregory, 2006; Maalki, 2002), instrumentalisées pour les besoins des ONG qui doivent - elles nous l'ont rappelé tout au long de la recherche - "plaire » au public québécois et canadien, dans un environnement de communication très compétitif et rébarbatif à des contenus trop graves et trop exigeants. Ce grand public est demandeur de sujets offrant un monde à l'image de ce qu'il pense connaître, un monde développé par et pour le profit. C'est ainsi qu'il consentira à se délester d'un peu de ce profit pour assurer une certaine reproduction à ce modèle. Qu'en pensent les bénéficiaires de l'aide? Ce même public ne le saura que minimalement, parfois simplement par quelques phrases retrouvées sur des messages écrits ou enregistrés relatant les bienfaits du développement. Les membres les plus engagés des publics de ces ONG ne sont pas dupes de ces dires tronqués et enchâssés dans de bien ingrats formats, connaisseurs qu'ils sont des codes de la camera obscura et des novlangues. Il semble ici non exagéré d'affirmer que ces messages relèvent d'une forme d'altérisation des bénéficiaires de l'aide: les individus et les populations des pays du Sud ne sont finalement " rencontrés " par ceux du Nord que lorsqu'ils sont semblables à eux, reflétant un très faible degré d'interculturalité, et cela malgré les idéaux altruistes qui traversent les organisations. Il est possible que ces messages, compte tenu de toutes les contraintes politiques évoquées par les ONG elles-mêmes, ne puissent complètement traduire les efforts de rencontre et de partage solidaire qui pourtant les caractérisent.

Une autre question est celle de la véracité du témoignage et de sa relation aux droits humains. Judith Butler (2007), dans son ouvrage Le récit de soi, rappelait l'importance du cadre de réception du témoignage et l'impossibilité de dire la vérité à partir de ce dernier. Un témoignage est toujours enchâssé dans un contexte élargi de réception qui en détermine la lisibilité. Malgré le caractère de preuve qu'il tend à prendre, même hors de la sphère juridique - et que la preuve soit une simple trace, un récit ou une performance - cette preuve par l'exemple de l'action réussie (comme on le fait dans les ONG) prend nécessairement un caractère culturel et politique, elle ne 
se suffit pas à elle-même. La définition que nous donnions du témoignage en début d'article nous permet de comprendre que ce dernier, hors de son application juridique, prend de plus en plus un caractère performatif et détextualisé, l'expansion du champ des communications aidant. Nous pourrions ajouter qu'il se confond de plus en plus dans sa forme (et de moins en moins dans son contenu) aux médias de masse. L'importance des images dans les messages et la place qu'elles occupent en incarnant la présence des bénéficiaires de l'aide, muets par ailleurs, en est la parfaite illustration. On peut dès lors se demander qui est au juste le témoin dans ces processus communicationnels. On l'a compris, ce témoin a une tête de Janus qui incorpore à la fois l'ONG et le bénéficiaire collectif de l'aide. Le témoignage est lancé dans l'espace public à un public qui devient, au bout du compte, celui qui jugera du tort subi. Le contenu du témoignage portera ainsi sur le tort subi, mais ce tort - ou si l'on préfère ces manquements aux droits ou leur précarité - n'apparaîtra qu'en arrière-plan, indirectement, partiellement déjà effacé par l'action de Janus. La vérité du tort subi a, tout au long de ce processus d'altérisation, subi l'épreuve du marketing.

\section{Références bibliographiques}

Beaudet, P. (2009). Qui aide qui? Une brève histoire de la coopération internationale au Québec. Montréal : Éditions du Boréal.

Blais, L. (2008). Vivre à la marge. Réflexions autour de la souffrance sociale. Québec: Presses de l'Université Laval.

Boltanski, L. (1993). La souffrance à distance. Paris : Métayer.

Butler, J. (2007). Le récit de soi. Paris, Presses Universitaires de France.

Charbonneau, F. (dir.). Le Québec au miroir de ses téléséries. Argument, 13(1), 50-75.

Chaumont, J.-M. (2002 [1997]). La concurrence des victimes. Paris : Payot.

Chiantaretto, J.-F. et Robin, R. (2003). Témoignages et écritures de l'histoire. Paris : L'Harmattan.

Coquio, C. (2003). L’histoire trouée. Négation et témoignage. Nantes: L'Atalante.

Conoir, Y., et Verna, G. (2002). L'action humanitaire du Canada. Québec: Presses de l’Université Laval.

Das, V., Kleinman, A., Lock, M., Ramphele, M. et Reynolds, P. (2001). Remaking a world. Violence, social suffering and recovery. Berkeley : University of California Press.

Das, V., Kleinman, A., Ramphele, M. et Reynolds, P. (2000). Violence and subjectivity. Berkeley : University of California Press.

Dauvin, P. et Siméant, J. (2002). Le travail humanitaire. Les acteurs des ONG du siège au terrain. Paris: Presses de Sciences Po.

Dulong, R. (1998). Le témoin oculaire. Les conditions sociales de l'attestation personnelle. Paris : Éditions de l'EHESS.

Erner, G. (2006). La société des victimes. Paris : La Découverte.

Ethridge, R. (2006). Bearing Witness : Assumptions, Realities and the Othering of Kathrina. American Anthropologist, 108(4), 799-813.

Fassin, D. (2004a). Et la souffrance devint sociale. De l'anthropologie médicale à une anthropologie des afflictions. Critique, 680-681, 16-29.

Fassin, D. (2004b). Des maux indicibles. Sociologie des lieux d'écoute. Paris : La Découverte.

Fassin, D. (2004c). La cause des victimes. Les temps modernes, 627, 73-91.

Fassin, D. (2006). L'humanitaire contre l’État. Tout contre. Vacarme, 34, 15-19.

Fassin, D. et Pandolfi, M. (2010). Contemporary states of emergency: the politics of military and humanitarian interventions. Cambridge : Zone.

Fassin D. et Rechtman, R. (2007). L'empire du traumatisme. Paris : Flammarion. 
Gregory, S. (2006). Transnational storytelling: human rights, witness, and video advocacy. American Anthropologist, 108(1), 195-204.

Hastrup, K. (2001a). Legal cultures and human rights. The challenge of diversity. The Hague : Kluwer.

Hastrup, K. (2001b). Human rights on common grounds. The quest for universality. The Hague : Kluwer.

Hastrup, K. (2003). Violence, suffering and human rights. Anthropological Theory, 3(3), 309-323.

Kilani, M. (2009). Figures de la preuve. Le cannibale et son témoin. Communications, 84, 45-58.

Kleinman, A., Das, V. et Lock, M. (1997). Social suffering. Berkeley : University of California Press.

Levi, P. (2005). CEuvres. Paris : Gallimard.

Levi, P. (2005 [1947]). Si c'est un homme (édition établie par Catherine Coquio). Paris : Robert Laffont.

Malkki, L. (1995). Refugees and exile : from "Refugees studies" to national order of things. Annual Review of Anthropology, 24, 495-523.

Maalki, L. (2002). Speechless Emissaries : Refugees, Humanitarianism and Deshistoricization. Dans A. Laban Hinton, Genocides (p. 244-367). Mailden : Blackwell.

McLagan, M. (2003). Human rights, testimony and transnational publicity. The Scholar and Feminist Online, 2(1). Récupéré du site de la revue : http://www.barnard.edu/sfonline/ps/printmmc.htm

Rancière, J. (2005). La haine de la démocratie. Paris : La Fabrique.

Ricœur, P. (2000). La mémoire, I'histoire, I’oubli. Paris : Seuil.

Saillant, F. (2006). Médias, Événement, Humanitaire. Dans I. Olazabal et J. Lévy, L'événement en anthropologie (p. 115-136). Québec: Presses de l’Université Laval.

Saillant, F. (dir.). (2007a). Entre-lieux de l’humanitaire. Anthropologie et sociétés, 31(2).

Saillant, F. (2007b). Handicaps et identités. La dignité pour horizon. Paris : Karthala.

Saillant, F. (2007c). "Vous êtes ici dans une mini-ONU" : les réfugiés publics au Québec. De I’humanitaire au communautaire. Anthropologie et Sociétés, 31(2), 65-90.

Saillant, F. (2011). Droits humains et témoignages : l'épreuve de la culture. Alterstice, 1(2), 3-8.

Saillant, F. et Boudreault-Fournier, A. (2011). Le témoignage dans les organisations de la société civile. DVD CÉLAT et ÉRASME.

Saillant, F., Paumier, M. et Richardson, M. (2005). L'Humanitaire et les identités : une perspective anthropologique. Ethnologies, 27(2), 160-187.

Saillant, F. et Truchon, K. (2008). Être plus que Corps. Lien social et Politiques, 59, 61-74.

Salmon, C. (2009). Storytelling. Paris : La Découverte.

Spivak, G. (2009). Les subalternes peuvent-elles parler? Paris : Éditions Amsterdam.

Todorov, T. (2004). Les abus de la mémoire. Paris : Arléa.

Wieviorka, A. (1998). L'ère du témoin. Paris : Plon. 